\title{
Inventário de Crenças de Carreira e Empregabilidade
}

\author{
Alexandra Barros ${ }^{1}$ \\ Faculdade de Psicologia, Universidade de Lisboa, Lisboa, Portugal
}

\section{RESUMO}

O presente trabalho reporta o desenvolvimento do Inventário de Crenças de Carreira e Empregabilidade que permite avaliar crenças facilitadoras de comportamentos e atitudes adaptativos dos indivíduos face a transições de carreira. O ICEB tem 38 itens e seis escalas: Esforço/Realização, Proatividade/Iniciativa, Flexibilidade/Abertura às mudanças, Aceitação de desafios/Riscos, Otimismo e Autonomia. São apresentadas as etapas de construção e os dados referentes às características psicométricas do instrumento, com uma amostra de 395 estudantes, 206 do sexo masculino e 189 do sexo feminino, entre os 18 e os 26 anos com média etária de 21,21 anos. Os índices de consistência interna e os indicadores de validade são satisfatórios, posicionando o ICEB como um instrumento promissor, podendo fundamentar intervenções vocacionais que visem facilitar transições na carreira.

Palavras-chave: crenças de carreira; empregabilidade; transições de carreira.

\section{ABSTRACT - Career and Employability Beliefs Inventory}

The present study reports the development of the Career and Employability Beliefs Inventory (Inventário de Crenças de Carreira e Empregabilidade - ICEB), which aims to evaluate beliefs that may correspond to the adaptive behaviors and attitudes of individuals facing career transitions. The ICEB has 38 items and 6 scales: Striving/Achievement, Proactivity/Initiative, Flexibility/Openness to change, Acceptance of challenges/Risks, Optimism, and Autonomy. We present the construction phases and report data regarding the psychometric characteristics of the instrument, studied with a sample of 395 students, 206 male and 189 female, with a mean age of 21.21 years. The internal consistency and validity indicators were satisfactory, presenting the ICEB as a promising instrument that supports the planning of vocational interventions aimed at facilitating career transitions.

Keywords: Career beliefs; Employability; Career transitions.

\section{RESUMEN - Inventario de Creencias de Carrera y Empleabilidad}

El presente trabajo reporta el desarrollo del Inventario de Creencias de Carrera y Empleabilidad (ICEB) que permite evaluar creencias facilitadoras que puedan corresponder a conductas y actitudes adaptativas de los individuos frente a transiciones de carrera. El ICEB dispone de 38 ítems y 6 escalas: Esfuerzo/Realización, Proactividad/Iniciativa, Flexibilidad/Apertura a los cambios, Aceptación de retos/Riesgos, Optimismo y Autonomía. Son presentadas las etapas de construcción y los datos referentes a las características psicométricas del instrumento, estudiado con una muestra de 395 estudiantes, 206 del sexo masculino y 189 del sexo femenino, con una media de edad de 21,21 años. Los índices de consistencia interna y los indicadores de validez son satisfactorios, posicionando al ICEB como un instrumento promisorio, pudiendo fundamentar intervenciones vocacionales que busquen facilitar transiciones en la carrera.

Palabras clave: Creencias de carrera; Empleabilidad; Transiciones de carrera.

Numa época marcada pela perceção da instabilidade e da imprevisibilidade do futuro, os percursos de carreira individuais deixaram de ser lineares e previsíveis, passando a caracterizar-se por inúmeras transições ao longo da vida (Barros, 2010; Chudzikowski, 2012). Deixou de haver uma única transição da escola ou da universidade para o trabalho mas várias transições durante a escolaridade, porque o indivíduo é chamado a fazer várias opções perante a flexibilidade do sistema de ensino, dentro do espírito de Bolonha, e durante toda a vida profissional.

O processo de adaptação ao contexto atual de um mundo globalizado e a possibilidade do indivíduo construir uma carreira satisfatória, lidando com as diferentes tarefas vocacionais e com a imprevisibilidade das mudanças que ocorrem no mundo do trabalho no atual contexto econômico pode envolver dimensões de empregabilidade (DiFabio, 2017; Fugate \& Kinicki, 2008; 
Fugate, Kinicki, \& Ashford, 2004), recursos de adaptabilidade (Hirschi, Herrmann, \& Keller, 2015; Rossier, 2015; Rossier, Ginevra, Bollmann, \& Nota, 2017; Savickas, 1997, 2005; Savickas \& Porfeli, 2012), mas também a disponibilidade para flexibilizar os objetivos individuais face à realidade (Van Esbroeck, 2008), e a capacidade para aproveitar e mesmo criar novas oportunidades (Krumboltz, 2009; Krumboltz, Foley, \& Cotter, 2013; Mitchell, Levin, \& Krumboltz, 1999). Apesar das influências contextuais reais/objetivas terem um papel importante nos percursos de carreira do indivíduo, este é o agente ativo na apreciação e atribuição de significado aos recursos existentes no seu ambiente/contexto (Lent, 2013; Lent, Brown, \& Hackett, 2002), mas também aos seus próprios recursos para gerir as transições de carreira nas diferentes fases da sua vida. Nesse sentido, os comportamentos adaptativos ou não adaptativos são mediados pelas crenças do indivíduo sobre si próprio, sobre o mundo e sobre o seu posicionamento no mundo. Essas crenças, que estão subjacentes à forma como ele utiliza as suas competências e se comporta, podem funcionar como facilitadoras ou como barreiras aos comportamentos de adaptação a contextos que exigem flexibilidade face à instabilidade e à mudança. Assim, quando as crenças dos indivíduos são consistentes com a proatividade, a iniciativa, o otimismo, a flexibilidade e a assunção da responsabilidade pela construção de um percurso satisfatório (Krumboltz, 2009; Krumboltz, Foley, \& Cotter, 2013; Krumboltz \& Levin, 2004; Mitchell et al., 1999; Tolentino et al., 2014) ou com autoperceções referentes às disposições pessoais geradoras de comportamentos adaptativos (Fugate \& Kinicki, 2008; Fugate, Kinicki, \& Ashford, 2004) ou com o domínio de recursos de adaptabilidade (Del Corso, 2017; Del Corso, Rehfuss, \& Galvin, 2011; Savickas et al., 2009, 2010/2011; Savickas, 2013) podem ser facilitadoras das transições de carreira e até contribuir para comportamentos que permitem aos indivíduos transformar acontecimentos não planeados ou acasos em oportunidades favoráveis (Krumboltz, 2009; Krumboltz et al., 2013; Krumboltz \& Levin, 2004). Pelo contrário, as crenças de que existem barreiras intransponíveis podem bloquear as ações mais adaptativas para a construção de um percurso de carreira satisfatório.

Considerando a conceção alargada dos objetivos do aconselhamento vocacional de Krumboltz e Chan (2005) e havendo evidência (e.g., Kovalski \& Horan, 1999) de que crenças de carreira disfuncionais ou menos adaptativas podem ser transformadas, a avaliação das crenças dos indivíduos sobre a carreira e a empregabilidade torna-se útil para o planeamento de intervenções vocacionais, que ajudem os clientes a desenvolver comportamentos e atitudes que favoreçam a sua proatividade e a construção do seu percurso de carreira. Tendo em conta essa necessidade de avaliação das crenças, a importância de integrar diferentes modelos e conceitos (Duarte, Silva,
\& Paixão, 2017) e o reconhecimento da forte influência dos contextos culturais na adaptabilidade (Rottingaus, Falk, \& Eshelman, 2017), foi criado um instrumento original, em língua portuguesa, adequado à atual realidade econômica e social: o ICEB: Inventário de Crenças de Carreira e Empregabilidade (Career and Employability Beliefs Inventory), suportado no modelo disposicional de empregabilidade (Fugate \& Kinicki, 2008; Fugate et al., 2004), no modelo de adaptabilidade (Savickas et al., 2009, 2010/2011; Savickas, 2013) e no modelo de casualidade planeada (Krumboltz, 2009).

Embora exista um instrumento designado Inventário de Crenças de Carreira (Career Beliefs Inventory) de Krumboltz (1991), com uma estrutura em 25 escalas, as suas escalas não avaliam apenas crenças ou esquemas, mas antes cinco grandes temas que se relacionam com motivações, atribuições, atitudes, intenções, valores, disponibilidade para mudanças geográficas, entre outros: "A minha situação atual; De que preciso para ser feliz; Fatores que influenciam as minhas decisões; Mudanças que estou disposto a fazer e Esforços que estou disposto a fazer". Essas escalas têm dimensões muito diferentes, sendo que dez delas só têm dois itens, o que contribui para coeficientes alfa de Cronbach muito baixos, levando alguns autores a considerar que a utilidade do inventário é principalmente a de ser "um estímulo para a discussão e autoexploração das estruturas cognitivas dos clientes" (Fuqua \& Newman, 1994, p. 430). Krumboltz (1991) situa o fundamento teórico desse instrumento como uma aplicação da terapia cognitiva (e.g., Beck, 1976; Ellis, 1967, citado por Krumboltz, 1991), permitindo que o conselheiro inclua no processo a exploração de atitudes e assunções que podem beneficiar de processos de reestruturação cognitiva. Outros instrumentos que avaliam variáveis relevantes para a gestão da carreira focam-se em modelos mais recentes, como o DME (Dispositional Measure of Employability, Fugate \& Kinicki, 2008), que permite avaliar as disposições de empregabilidade ou como a Career Adapt-Abilities Scale (CAAS) (Savickas \& Porfeli, 2012), que avalia as dimensões da adaptabilidade.

O ICEB procura integrar diferentes modelos e centra-se nas crenças de carreira facilitadoras ou inibidoras subjacentes aos comportamentos e às respostas adaptativas exigidos aos jovens nas múltiplas transições de carreira com que se vão deparar, incluindo a transição da escola para o mercado de trabalho. O pressuposto é que, sendo as crenças e as autoperceções do indivíduo sobre as suas competências e recursos (acerca das várias dimensões da adaptabilidade e da empregabilidade) e as suas perceções sobre as exigências do exterior que orientam a tendência de resposta do indivíduo face às escolhas e às transições com que se confronta, é nessas variáveis que se deve focar a avaliação. O presente trabalho visa descrever a metodologia de construção da prova e a análise das suas características psicométricas. 


\section{Método}

\section{Participantes}

A versão final do ICEB foi aplicada a 395 estudantes de escolas portuguesas, 206 (52,2\%) do sexo masculino e $189(47,8 \%)$ do sexo feminino, entre os 18 e os 26 anos com média etária de 21,21 e desvio padrão de 2,25, no final da escolaridade obrigatória $\left(12^{\circ}\right.$ ano de escolaridade) ou a frequentar o ensino superior.

\section{Instrumento}

Desenvolvimento do Instrumento. Para garantir um enquadramento integrador de modelos teóricos de referência sobre adaptabilidade, empregabilidade e mediação cognitiva, partiu-se de uma revisão bibliográfica sobre o modelo de Casualidade Planeada (Happenstance Learning Theory, HLT; Krumboltz, 2009), o modelo de adaptabilidade (Savickas \& Porfeli, 2012) e o modelo de empregabilidade (Fugate \& Kinicki, 2008). Para assegurar ainda que as crenças avaliadas representassem as atitudes e comportamentos valorizados pelos empregadores na contratação de novos elementos ou na decisão de promovê-los, foram conduzidas 15 entrevistas semiestruturadas a gestores de pequenas/médias empresas. Alguns exemplos de questões formuladas "quando avalia um potencial candidato para a sua empresa, que características procura?", "Que atitudes face ao trabalho valoriza nos trabalhadores?", "Descreva o trabalhador ideal para lidar com a atual instabilidade do mercado", "Considerando a sua experiência e as mudanças por que tem passado no seu próprio percurso profissional, que competências e comportamentos the parecem mais importantes para lidar com a mudança?", "Na sua empresa, quais os fatores determinantes para a progressão profissional?".

A análise de conteúdo feita por dois juizes independentes, psicólogos, permitiu identificar categorias que representam características pessoais relacionadas com crenças e atitudes face ao trabalho. Analisou-se a coincidência dessas categorias com as emergentes da revisão de literatura e foram escolhidas seis dimensões para a construção do inventário: Esforço/Realização, Proatividade/ Iniciativa, Flexibilidade/Abertura às mudanças, Aceitação de desafios/Riscos, Otimismo e Autonomia. Três psicólogos escreveram, separadamente, três itens para avaliar cada uma dessas seis dimensões e a totalidade dos 54 itens assim construídos foi aplicada a 38 estudantes universitários, para se avaliar a sua clareza e adequação. A partir dessa aplicação foram feitas algumas alterações à redação dos itens. Posteriormente, para selecionar os itens que otimizariam as propriedades psicométricas do instrumento, o inventário foi aplicado a 235 estudantes universitários, com idades entre os 18 e os 26 anos $(M=19,5$; $D P=1,85), 93$ participantes $(39,6 \%)$ do sexo masculino e $142(60,4 \%)$ do sexo feminino. Os estudantes deveriam classificar numa escala de 1 a 5 , de 1 - Discordo totalmente, a 5 - Concordo totalmente, o seu grau de concordância com cada uma das afirmações contidas nos itens. Com base nos dados obtidos, foram retirados os itens que não revelaram capacidade de discriminação ou cuja omissão beneficiava o alfa de Cronbach da escala a que pertence.

Versão Final do Instrumento. A versão final do ICEB (Inventário de Crenças de Carreira e Empregabilidade - Career and Employment Beliefs Inventory) é constituída por 38 itens e seis escalas, assumindo-se a definição de crenças de carreira de Krumboltz (1994). A escala Esforço/Realização inclui seis itens e avalia o grau de acordo do indivíduo com afirmações sobre a importância do esforço, da persistência e da resiliência para atingir objetivos de carreira. A escala Proatividade/ Iniciativa inclui oito itens e avalia o grau de acordo do indivíduo com afirmações sobre a importância das suas próprias ações no sentido de aproveitar ou criar oportunidades de trabalho. A escala Flexibilidade/Abertura às mudanças inclui sete itens e avalia o grau de acordo do indivíduo com afirmações sobre a sua disponibilidade para flexibilizar os seus objetivos e para se adaptar a diferentes situações e mudanças. A escala Aceitação de desafios/Riscos inclui cinco itens e avalia o grau de acordo do indivíduo com afirmações sobre a sua disponibilidade para correr riscos e aceitar desafios, encarando-os como oportunidades de desenvolvimento pessoal e profissional. A escala Otimismo inclui sete itens e avalia o grau de acordo do indivíduo com afirmações que refletem expectativas positivas face ao seu futuro. A escala Autonomia inclui cinco itens e avalia o grau de acordo do indivíduo com afirmações sobre a sua capacidade para desenvolver uma autonomia crescente no trabalho.

O inventário tem o formato de uma série de afirmações em relação às quais os participantes devem indicar, numa escala tipo Likert de 5 pontos (desde "discordo totalmente" até "concordo totalmente") o seu grau de acordo com a afirmação enunciada. Os itens que são formulados de forma inversa, representando o polo negativo de cada escala, são recodificados de forma que, em todos os casos, um resultado maior no total da escala represente maior grau de acordo com crenças facilitadoras da adaptação a transições de carreira.

\section{Procedimento}

Este estudo cumpre todos os princípios deontológicos e éticos definidos pela Ordem dos Psicólogos Portugueses e foi aprovado pela Comissão de Deontologia da Faculdade de Psicologia da Universidade de Lisboa. O estudo das características psicométricas do instrumento foi feito com base em indicadores de precisão e de validade. A análise da precisão dos resultados focou-se no estudo da consistência interna, com base nos coeficientes alfa de Cronbach, nas correlações item-total excluindo o item e no comportamento do alfa de Cronbach perante a omissão de cada item. Foi ainda realizada uma análise em componentes principais, com scree test, seguida de rotação varimax da matriz. No sentido de contribuir para a 
validação do ICEB - Inventário de Crenças de Carreira e Empregabilidade foi também aplicada a adaptação portuguesa do DME - Dispositional Measure of Employability. A adaptação portuguesa do DME (Fraga, 2012) tem cinco escalas: Abertura a mudanças no trabalho que avaliam o grau em que os indivíduos são recetivos ou desejosos da mudança, considerando que as mudanças são, em geral, positivas; Proatividade no trabalho e na carreira, que avalia uma orientação proativa na carreira e reflete as tendências e ações dos indivíduos para obterem informação que potencialmente afete as suas oportunidades de carreira; Motivação na carreira, que avalia o grau em que os indivíduos tendem a formular planos e estratégias de carreira específicos e a estabelecer objetivos relacionados com o trabalho/carreira; Resiliência no trabalho e na carreira, que avalia o grau em que os indivíduos são otimistas acerca das suas oportunidades de carreira e do trabalho e sentem que têm controle sobre as suas carreiras; e Identidade no trabalho, que avalia o grau em que os indivíduos se definem a si mesmos a partir de uma organização, emprego, profissão ou área de atividade em particular.

\section{Resultados}

A análise da consistência interna revela coeficientes alfa de Cronbach satisfatórios, variando entre 0,68 e 0,86: escala Esforço/Realização (0,86), escala Proatividade/Iniciativa $(0,68)$, escala Flexibilidade/ Abertura às mudanças $(0,71)$, escala Aceitação de desafios/Riscos $(0,81)$, escala Otimismo $(0,72)$ e escala Autonomia $(0,77)$. As correlações item-total (se o item for omitido para evitar relações espúrias) são também muito satisfatórias (Tabela 1).

Tabela 1

Coeficientes Alfa de Cronbach dos Totais das Escalas do ICEB

\begin{tabular}{|c|c|c|c|c|c|}
\hline \multicolumn{3}{|c|}{$\begin{array}{l}\text { Escala Flexibilidade/Adaptação mudanças: } \\
\text { coeficiente alfa de Cronbach=0,71 }\end{array}$} & \multicolumn{2}{|c|}{$\begin{array}{l}\text { Escala Otimismo: coeficiente } \\
\text { alfa de Cronbach }=0,72\end{array}$} & \multirow{2}{*}{$\begin{array}{l}\text { Alfa de } \\
\text { Cronbach se } \\
\text { item omisso }\end{array}$} \\
\hline item & $\begin{array}{c}\text { Correlação } \\
\text { item-total } \\
\text { corrigida }\end{array}$ & $\begin{array}{l}\text { Alfa de Cronbach } \\
\text { se item omisso }\end{array}$ & item & $\begin{array}{c}\text { Correlação } \\
\text { item-total } \\
\text { corrigida }\end{array}$ & \\
\hline 1 & 0,53 & 0,65 & 3 & 0,36 & 0,71 \\
\hline 2 & 0,42 & 0,67 & 4 & 0,53 & 0,67 \\
\hline 5 & 0,46 & 0,66 & 16 & 0,32 & 0,72 \\
\hline 12 & 0,29 & 0,71 & 20 & 0,49 & 0,68 \\
\hline 24 & 0,50 & 0,66 & 26 & 0,46 & 0,68 \\
\hline 32 & 0,41 & 0,68 & 30 & 0,52 & 0,67 \\
\hline 35 & 0,34 & 0,69 & 34 & 0,37 & 0,71 \\
\hline \multicolumn{3}{|c|}{$\begin{array}{l}\text { Escala Aceitação de desafios/Riscos: } \\
\text { coeficiente alfa de Cronbach=0,81 }\end{array}$} & \multicolumn{2}{|c|}{$\begin{array}{c}\text { Escala Autonomia: } \\
\text { coeficiente alfa de Cronbach }=0,77\end{array}$} & \\
\hline item & $\begin{array}{c}\text { Correlação } \\
\text { item-total } \\
\text { corrigida }\end{array}$ & $\begin{array}{l}\text { Alfa de Cronbach } \\
\text { se item omisso }\end{array}$ & item & $\begin{array}{c}\text { Correlação } \\
\text { item-total } \\
\text { corrigida } \\
\end{array}$ & $\begin{array}{c}\text { Alfa de } \\
\text { Cronbach se } \\
\text { item omisso }\end{array}$ \\
\hline 10 & 0,61 & 0,76 & 11 & 0,57 & 0,72 \\
\hline 15 & 0,66 & 0,75 & 19 & 0,51 & 0,74 \\
\hline 18 & 0,58 & 0,77 & 27 & 0,50 & 0,74 \\
\hline 28 & 0,60 & 0,77 & 37 & 0,59 & 0,71 \\
\hline \multirow[t]{2}{*}{36} & 0,53 & 0,79 & 38 & 0,55 & 0,73 \\
\hline & \multicolumn{2}{|c|}{$\begin{array}{c}\text { Escala Esforço/Realização: } \\
\text { coeficiente alfa de Cronbach=0,86 }\end{array}$} & \multicolumn{2}{|c|}{$\begin{array}{l}\text { Escala Proatividade/Iniciativa: } \\
\text { coeficiente alfa de Cronbach=0,68 }\end{array}$} & \\
\hline item & $\begin{array}{l}\text { Correlação item- } \\
\text { total corrigida }\end{array}$ & $\begin{array}{l}\text { Alfa de Cronbach } \\
\text { se item omisso }\end{array}$ & item & $\begin{array}{c}\text { Correlação } \\
\text { item-total } \\
\text { corrigida }\end{array}$ & $\begin{array}{c}\text { Alfa de } \\
\text { Cronbach se } \\
\text { item omisso }\end{array}$ \\
\hline 8 & 0,62 & 0,84 & 6 & 0,47 & 0,63 \\
\hline 13 & 0,69 & 0,82 & 7 & 0,52 & 0,61 \\
\hline 17 & 0,60 & 0,84 & 9 & 0,35 & 0,66 \\
\hline 21 & 0,63 & 0,84 & 14 & 0,28 & 0,67 \\
\hline 29 & 0,70 & 0,82 & 22 & 0,30 & 0,67 \\
\hline \multirow[t]{3}{*}{33} & 0,62 & 0,84 & 23 & 0,32 & 0,66 \\
\hline & & & 25 & 0,39 & 0,65 \\
\hline & & & 31 & 0,35 & 0,66 \\
\hline
\end{tabular}


A análise da Tabela 1 revela que as correlações de cada item com o total corrigido (excluindo o próprio item) da escala a que pertence variam entre 0,28 e 0,70 mas são quase todas superiores a 0,30. Além disso, a omissão de cada um dos itens, afetaria negativamente o valor do coeficiente alfa de Cronbach da escala a que pertence.

Foi também realizada uma análise em componentes principais aos itens, tendo, após o scree test, sido estudado um modelo em quatro fatores que, no seu conjunto, explicam 41,84\% da variância dos dados. A Tabela 2 apresenta as saturações dos itens em cada fator, os eigenvalues e a variância explicada por cada fator. São apresentadas a negrito as saturações mais elevadas em cada fator, verificando-se que no fator 1 saturam com valores mais altos os itens das escalas Aceitação de Desafios/Riscos e Flexibilidade/ Abertura às Mudanças, no fator 2 os itens da escala Esforço/ Realização, no fator 3 os itens da escala Otimismo e no fator 4 os itens da escala Proatividade/Iniciativa. Os itens da escala Autonomia aparecem com maiores saturações nos fatores 1 e 2. Assim, embora a estrutura do inventário não seja completamente replicada a partir da análise fatorial, os dados apontam para uma estrutura interna que se aproxima do modelo que serviu de base à construção das escalas.

Tabela 2

Análise Fatorial dos Itens do ICEB (N=395)

\begin{tabular}{|c|c|c|c|c|}
\hline Item & Fator 1 & Fator 2 & Fator 3 & Fator 4 \\
\hline Item 8 & 0,42 & 0,60 & 0,01 & 0,11 \\
\hline Item 13 & 0,22 & 0,70 & 0,12 & 0,11 \\
\hline Item 17 & 0,37 & 0,59 & 0,07 & 0,06 \\
\hline Item 21 & 0,05 & 0,78 & 0,10 & 0,05 \\
\hline Item 29 & 0,11 & 0,72 & 0,23 & 0,04 \\
\hline Item 33 & 0,30 & 0,59 & 0,20 & 0,01 \\
\hline Item 10 & 0,61 & 0,22 & 0,05 & 0,12 \\
\hline item 15 & 0,63 & 0,24 & 0,06 & 0,13 \\
\hline Item 18 & 0,61 & 0,12 & $-0,03$ & 0,24 \\
\hline Item 28 & 0,65 & 0,29 & 0,11 & 0,01 \\
\hline Item 36 & 0,72 & 0,04 & 0,15 & 0,07 \\
\hline Item 3 & 0,18 & 0,30 & 0,38 & $-0,06$ \\
\hline Item 4 & 0,22 & 0,28 & 0,60 & $-0,04$ \\
\hline Item 16 & $-0,01$ & $-0,12$ & 0,63 & 0,15 \\
\hline Item 20 & 0,05 & 0,04 & 0,70 & 0,1 \\
\hline Item 26 & $-0,09$ & 0,25 & 0,58 & 0,10 \\
\hline Item 30 & 0,12 & 0,25 & 0,58 & 0,03 \\
\hline Item 34 & 0,01 & 0,21 & 0,44 & 0,03 \\
\hline Item 6 & 0,14 & 0,16 & $-0,10$ & 0,65 \\
\hline Item 7 & 0,04 & 0,05 & $-0,01$ & 0,74 \\
\hline Item 9 & $-0,12$ & 0,15 & 0,03 & 0,57 \\
\hline Item 14 & 0,15 & 0,05 & 0,18 & 0,35 \\
\hline Item 22 & 0,08 & $-0,04$ & 0,31 & 0,41 \\
\hline Item 23 & 0,11 & $-0,07$ & 0,28 & 0,42 \\
\hline Item 25 & $-0,03$ & 0,25 & $-0,01$ & 0,57 \\
\hline Item 31 & 0,17 & $-0,06$ & 0,01 & 0,54 \\
\hline Item 11 & 0,29 & 0,63 & 0,14 & 0,14 \\
\hline Item 19 & 0,28 & 0,58 & 0,15 & 0,15 \\
\hline Item 27 & 0,54 & 0,38 & 0,09 & $-0,01$ \\
\hline Item 37 & 0,63 & 0,31 & $-0,01$ & 0,09 \\
\hline Item 38 & 0,48 & 0,34 & 0,09 & 0,03 \\
\hline Item 1 & 0,47 & 0,41 & $-0,02$ & 0,14 \\
\hline Item 2 & 0,32 & 0,48 & 0,07 & 0,10 \\
\hline Item 5 & 0,54 & 0,09 & $-0,03$ & 0,07 \\
\hline Item 12 & 0,23 & 0,14 & $-0,21$ & 0,07 \\
\hline Item 24 & 0,53 & 0,28 & 0,02 & $-0,03$ \\
\hline
\end{tabular}


Tabela 2 (continuação)

Análise Fatorial dos Itens do ICEB ( $N=395)$

\begin{tabular}{|c|c|c|c|c|}
\hline Item & Fator 1 & Fator 2 & Fator 3 & Fator 4 \\
\hline Item 32 & 0,59 & 0,11 & 0,17 & 0,05 \\
\hline Item 35 & 0,62 & $-0,01$ & $-0,01$ & $-0,04$ \\
\hline Eigenvalues & 9,18 & 2,68 & 2,251 & 1,782 \\
\hline \% Variância explicada & 24,16 & 7,06 & 5,92 & 4,69 \\
\hline
\end{tabular}

A análise das correlações entre as escalas ICEB (Tabela 3) revela coeficientes acima de 0,50 entre Esforço/ Realização com Autonomia $(0,69)$, com Flexibilidade/ Abertura às Mudanças $(0,50)$ e com Aceitação de desafios/Riscos (0,52), entre Flexibilidade/Abertura às
Mudanças com Aceitação de desafios/Riscos $(0,63)$ e com Autonomia $(0,64)$, e entre Aceitação de desafios/ Riscos com Autonomia $(0,63)$. Todas as correlações entre as escalas são iguais ou superiores a 0,20 e são todas significativas a $p<0,01$.

Tabela 3

Correlações entre as Escalas ICEB (N=395)

\begin{tabular}{|c|c|c|c|c|c|}
\hline & $\begin{array}{l}\text { Proatividade/ } \\
\text { Iniciativa }\end{array}$ & $\begin{array}{c}\text { Flexibilidade } \\
\text { Adaptação } \\
\text { mudanças }\end{array}$ & $\begin{array}{c}\text { Aceitação de } \\
\text { desafios/Riscos }\end{array}$ & Otimismo & Autonomia \\
\hline Esforço/Realização & $0,25^{* *}$ & $0,50^{* *}$ & $0,52^{* *}$ & $0,39^{* *}$ & $0,69^{* *}$ \\
\hline Proatividade/Iniciativa & & $0,20^{* *}$ & $0,26^{* *}$ & $0,23^{* *}$ & $0,27^{* *}$ \\
\hline Flexibilidade/Adaptação mudanças & & & $0,63^{* *}$ & $0,24^{* *}$ & $0,64^{* *}$ \\
\hline Aceitação de desafios/Riscos & & & & $0,25^{* *}$ & $0,63^{* *}$ \\
\hline Otimismo & & & & & $0,34^{* *}$ \\
\hline
\end{tabular}

Nota. ${ }^{*} p<0,05 ;{ }^{* *} p<0,01$

A análise dos coeficientes de correlação de Pearson entre as escalas do ICEB e as escalas do DME (Tabela 4) permite contribuir para a sua validação. A escala de Esforço/Realização apresenta correlações positivas, superiores a 0,5 e significativas a $p<0,01$ com Motivação na carreira $(0,68)$ e Resiliência $(0,63)$. A escala de Proatividade/Iniciativa do ICEB apresenta correlações positivas e significativas com todas as escalas da DME, mas a mais elevada é de 0,5 precisamente com a escala de Proatividade no trabalho e na carreira do DME. A escala
Flexibilidade/Abertura às mudanças do ICEB também apresenta correlações positivas e significativas a $p<0,01$, com todas as escalas do DME, mas a mais expressiva é com a escala Abertura a mudanças no trabalho do DME $(0,6)$. Otimismo, Autonomia e Aceitação de desafios/ Riscos também apresentam correlações positivas e significativamente estatísticas com todas as escalas do DME, salientando-se a correlação de 0,61 entre Identidade e Autonomia, as duas escalas mais relacionadas com a expressão individual.

Tabela 4

Correlações entre as Escalas ICEB e DME (N=395)

\begin{tabular}{lccccc}
\hline \multicolumn{1}{c}{ ICEB /DME } & Abertura & Proatividade & Motivação & Resiliência & Identidade \\
\hline Esforço/Realização & $0,31^{* *}$ & $0,15^{* *}$ & $0,68^{* *}$ & $0,63^{* *}$ & $0,44^{* *}$ \\
Proatividade/Iniciativa & $0,10^{*}$ & $0,50^{* *}$ & $0,16^{* *}$ & $0,14^{* *}$ & $0,13^{* *}$ \\
Flexibilidade/Adaptação mudanças & $0,60^{* *}$ & $0,15^{* *}$ & $0,32^{* *}$ & $0,35^{* *}$ & 0,14 \\
Aceitação de desafios/Riscos & $0,40^{* *}$ & $0,17^{* *}$ & $0,38^{* *}$ & $0,42^{* *}$ & $0,31^{* *}$ \\
Otimismo & $0,16^{* *}$ & $0,13^{* *}$ & $0,35^{* *}$ & $0,32^{* *}$ & $0,24^{* *}$ \\
Autonomia & $0,44^{* *}$ & $0,16^{* *}$ & $0,48^{* *}$ & $0,44^{* *}$ & $0,61^{* *}$ \\
\hline
\end{tabular}

Nota. ${ }^{*} p<0,05 ;{ }^{* *} p<0,01$ 
O teste $t$ de diferença de médias para amostras independentes revela médias significativamente superiores para as mulheres $(a p<0,01)$ apenas nas escalas de
Flexibilidade/Abertura às mudanças e Aceitação de desafios/Riscos. Nas outras escalas, não se encontram diferenças significativas entre sexos (Tabela 5).

Tabela 5

Teste t Student para Amostras Independentes para Diferenças entre Gêneros

\begin{tabular}{|c|c|c|c|c|c|c|}
\hline Escalas ICEB & $\mathrm{N}$ & Sexo & Média & $\mathrm{DP}$ & $t$ & $p$ \\
\hline \multirow{2}{*}{ Esforço/Realização } & 189 & Feminino & 4,36 & 0,64 & & \\
\hline & 206 & Masculino & 4,29 & 0,68 & $-10,02$ & 0,31 \\
\hline \multirow{2}{*}{ Proatividade/Iniciativa } & 189 & Feminino & 3,79 & 0,50 & $-0,8$ & 0,42 \\
\hline & 206 & Masculino & 3,76 & 0,53 & & \\
\hline \multirow{2}{*}{ Flexibilidade/Adaptação Mudanças } & 189 & Feminino & 4,19 & 0,41 & $-30,43$ & 0,001 \\
\hline & 206 & Masculino & 4,01 & 0,61 & & \\
\hline \multirow{2}{*}{ Aceitação de desafios/Riscos } & 189 & Feminino & 4,34 & 0,46 & $-20,9$ & 0,004 \\
\hline & 206 & Masculino & 4,18 & 0,60 & & \\
\hline \multirow{2}{*}{ Otimismo } & 189 & Feminino & 3,55 & 0,68 & & \\
\hline & 206 & Masculino & 3,59 & 0,62 & 0,65 & 0,52 \\
\hline \multirow{2}{*}{ Autonomia } & 189 & Feminino & 4,44 & 0,70 & & \\
\hline & 206 & Masculino & 4,36 & 0,70 & 1,5 & 0,14 \\
\hline
\end{tabular}

Nota. ${ }^{*} p<0,05 ;{ }^{* *} p<0,01$

\section{Discussão}

A análise aos dados obtidos com o ICEB revela que este possui propriedades metrológicas adequadas, posicionando-se como um instrumento promissor para avaliar o conjunto de crenças de estudantes e trabalhadores sobre os comportamentos adequados para a adaptação às exigências atuais do mundo do trabalho e sobre os seus próprios recursos para lidarem com as transições de carreira com que se irão deparar. As subescalas do ICEB revelam boa consistência interna com correlações item-total e valores do coeficiente alfa de Cronbach adequados. A análise fatorial aos itens permite reproduzir parcialmente a estrutura do instrumento, com os itens de cada escala a saturarem no mesmo fator. Os indicadores de validade referentes ao cruzamento com um instrumento de avaliação das disposições para a Empregabilidade: DME (Fraga, 2012), já adaptado para a população portuguesa, revelam que as dimensões avaliadas pelos dois instrumentos (ICEB e DME) estão correlacionadas positiva e significativamente. A qualidade de construção desse instrumento permite, assim, avaliar, com rigor, se os indivíduos possuem um conjunto de crenças facilitadoras das transições de carreira ou, se pelo contrário, as suas crenças de carreira se constituem como barreiras que podem dificultar a construção de um percurso de carreira satisfatório.

Considerando que cada vez mais é o indivíduo que tem a responsabilidade de construir o seu percurso de vida, numa época em que o percurso de carreira já não se faz de forma linear com uma única transição da escola para o trabalho e toda a vida profissional num emprego estável, os indivíduos, necessitam de manter uma empregabilidade flexível, por meio da aprendizagem ao longo da vida e do desenvolvimento das suas competências (Amundson, Mills, \& Smith, 2014). De fato, o contexto atual, caracterizado pela perceção de instabilidade e de imprevisibilidade do futuro, mas também de oportunidades de mobilidade em função de uma economia e de um mundo globalizado, implica a agência do indivíduo e é nesse sentido que as características disposicionais, as competências e as atitudes que facilitam os comportamentos adaptativos face às transições de carreira adquirem especial importância (Hirschi et al., 2015; Rossier et al., 2017). Admitindo que as tomadas de decisão de carreira serão frequentes, as intervenções vocacionais podem, assim, ser fundamentais para preparar os indivíduos para as transições de carreira, de forma a ajudá-los a desenvolver um mindset alinhado com as necessidades atuais do mundo do trabalho. Sendo os comportamentos e as intenções mediados pelas crenças, as conceções adaptativas que os indivíduos têm sobre si próprios e sobre a carreira podem facilitar as suas tomadas de decisão, influenciando a sua forma de lidar com as transições de carreira e levando a escolhas mais satisfatórias e viáveis (Bandura, 1997; 2001; Hirschi et al., 2015; Krumboltz \& Levin, 2004).

Nessa perspetiva, a expansão do conceito de alvo das intervenções, visando a mudança dos indivíduos e 
do meio, por meio de técnicas cognitivas e comportamentais adquire especial relevância, permitindo enquadrar a preparação dos indivíduos para explorarem novos caminhos ou novas áreas de interesse, para manterem o esforço apesar de algumas barreiras, para flexibilizar objetivos e serem capazes de correr riscos e aceitar desafios mesmo em contextos de incerteza e serem capazes de reconhecer, aproveitar e mesmo provocar a ocorrência de acontecimentos inesperados enquanto circunstâncias favoráveis (Krumboltz, 2009; Krumboltz \& Levin, 2004). É assim relevante a avaliação das crenças para caracterizar as necessidades de intervenção de indivíduos e de grupos, de modo a poder ajudá-los a desenvolver um sistema de crenças compatível com os recursos pessoais que promovem os comportamentos adaptativos face às mudanças. Com base nessa avaliação, que pode ser proporcionada pelo ICEB, o aconselhamento de carreira pode desenhar intervenções vocacionais, individualizadas ou em grupo, que promovam e fomentem crenças que favoreçam a flexibilidade, a empregabilidade e a adaptabilidade quando os indivíduos enfrentam transições normativas ou não normativas na carreira.

O presente estudo foi realizado com recurso a amostras de conveniência, o que constitui uma limitação. A exploração da versão final do instrumento foi baseada numa amostra de dimensão razoável, permitindo concluir da adequação das características metrológicas do ICEB com essa população de jovens adultos. No entanto, serão necessários estudos subsequentes, com amostras maiores e abrangendo indivíduos em várias faixas etárias e em outros momentos do seu percurso escolar e profissional, para melhor explorar as potencialidades desse instrumento. Estudos posteriores também poderão basear-se em amostras diferenciadas a partir de variáveis como a área de estudos ou área profissional, ou a área de residência (litoral ou interior, por exemplo), na medida que, a essas variáveis, correspondem diferentes perspetivas de colocação no mercado de trabalho.

\section{Referências}

Amundson, N., Mills, L., \& Smith, B. (2014). Incorporating chaos and paradox into career development. Australian Journal of Career Development, 23(1), 13-21. doi: 10.1177/1038416213496760

Bandura, A. (1997). Self-efficacy: The exercise of control. New York: Freeman.

Bandura, A. (2001). Social cognitive theory: An Agentic perspective. Annual Review of Psychology, 52(1), 1-26. doi: 10.1146/annurev.psych.52.1.1

Barros, A. (2010). Desafios da Psicologia Vocacional: Modelos e intervenções na era da incerteza. Revista Brasileira de Orientação Profissional, $11(2), 165-175$.

Chudzikowski, K. (2012). Career transitions and career success in the "new" career era. Journal of Vocational Behavior, 81(2), 298-306. doi: 10.1016/j.jvb.2011.10.005

Del Corso, J. J. (2017). Counseling young adults to become career adaptable and career resilient. Em K. Maree (Ed). Psychology of Career Adaptability, Employability and Resilience (pp.171-188). Springer International Publishing.

Del Corso, J. J., Rehfuss, M. C., \& Galvin, K. (2011). Striving to adapt: Addressing Adler's work task in the 21st century. The Journal of Individual Psychology, 67(2), 88-106.

Di Fabio, A. (2017). A Review of Empirical Studies of Employability and Measures of Employability. Em K. Maree (Ed). Psychology of Career Adaptability, Employability and Resilience (pp.107-123). Springer International Publishing.

Duarte, M. E., Silva, J. T., \& Paixão, M. P. (2017). Career Adaptability, Employability and Career Resilience in Managing Transitions. Em K. Maree (Ed). Psychology of Career Adaptability, Employability and Resilience (pp. 241-261). Springer International Publishing.

Fraga, S. (2012). Adaptabilidade e empregabilidade numa perspectiva construtivista (Tese de Doutorado). Faculdade de Psiologia, Universidade de Lisboa, Lisboa.

Fugate, M., \& Kiniki, A. J. (2008). A dispositional approach to employability: Development of a measure and test of implications for employee reactions to organizational change. Journal of Occupational and Organizational Psychology, 81(3), 503-527. doi: 10.1348/096317907X241579

Fugate, M., Kinicki, A. J., \& Ashforth, B. E. (2004). Employability: A psycho-social construct, its dimensions, and applications. Journal of Vocational Behavior, 65, 14-38.

Fuqua, D. R., \& Newman, J.L. (1994). An Evaluation of the Career Beliefs Inventory. Journal of Counseling and Development, 72(4), 429-430. doi: 10.1002/j.1556-6676.1994.tb00963.x

Hirschi, A., Herrmann, A., \& Keller, A. (2015). Career adaptivity, adaptability, and adapting: A conceptual and empirical investigation. Journal of Vocational Behavior, 87, 1-10. doi: 10.1016/j.jvb.2014.11.008

Kovalski, T. M., \& Horan, J. J. (1999). The effects of Internetbased cognitive restructuring on the irrational career beliefs of adolescent girls. Journal of Cognitive Psychotherapy, 13(2), 145-152. doi: 10.1891/0889-8391.13.2.145

Krumboltz, J. D. (1991). Manual for the Career Beliefs Inventory. Palo Alto: Consulting Psychologist Press.

Krumboltz, J. D. (1994). The Career Beliefs Inventory. Journal of Counseling \& Development, 72(4), 424-428. doi: 10.1002/j.1556-6676.1994. tb0096

Krumboltz, J. D. (2009). The Happenstance Learning Theory. Journal of Career Assessment, 17(2), 135-154. doi: 10.1177/1069072708328861

Krumboltz, J. D., \& Chan, A. (2005). Professional issues in Vocational Psychology. Em W. B. Walsh \& M. L. Savickas (Eds.), Handbook of Vocational Psychology: Theory, Research and Practice (3rd edition, pp. 347-369). London: Lawrence Erlbaum Associates.

Krumboltz, J. D., \& Levin, A. S. (2004). Luck is no accident: Making the most of happenstance in your life and career. Atascadero, CA: Impact.

Krumboltz, J. D., Foley, P. F., \& Cotter, E. W. (2013). Applying the happenstance learning theory to involuntary career transitions. The Career Development Quarterly, 61(1), 15-26. doi: 10.1002/j.2161-0045.2013.00032.x 
Lent, R. W. (2013). Social cognitive career theory. Em S. D. Brown \& R. W. Lent (Eds.), Career development and counseling: Putting theory and research to work (2nd ed., pp. 115-146). Hoboken, NJ: Wiley.

Lent, R. W., Brown, S. D., \& Hackett, G. (2002). Social cognitive career theory. Em D. Brown, \& Associates (Eds.), Career choice and development (4th ed., pp. 255-311). San Francisco: Jossey-Bass.

Mitchell, L., Levin, A. S., \& Krumboltz, J. D. (1999). Planned happenstance: Constructing unexpected career opportunities. Journal of Counseling Development, 77(2), 115-124. doi: 10.1002/j.1556-6676.1999.tb02431.x

Rossier, J. (2015). Career adaptability and life designing. Em L. Nota \& J. Rossier (Eds.). Handbook of life design: From practice to theory and from theory to practice (pp. 153-167). Boston, MA: Hogrefe Publishing.

Rossier, J., Ginevra, M.C., Bollmann, G., \& Nota. L. (2017). The importance of career adaptability, career resilience and employability in designing a successful life. Em K. Maree (Ed). Psychology of Career Adaptability, Employability and Resilience (pp.65-84). Springer International Publishing.

Rottingaus, P. J., Falk, N. A., \& Eshelman, A. (2017). Assessing Career Adaptability. Em K. Maree (Ed). Psychology of Career Adaptability, Employability and Resilience (pp. 85-105). Springer International Publishing.

Savickas, M. L. (1997). Career adaptability: An integrative construct for life-span, life-space theory. Career Development Quartely, 45, $247-259$. doi: 10.1002/j.2161-0045.1997.tb00469.x

Savickas, M. L. (2005). The theory and practice of career construction. In D. Brown \& R. W. Lent (Eds.), Career development and counseling: Putting theory and research to work (pp. 42-70). Hoboken, NJ: John Wiley \& Sons.

Savickas, M. L. (2013). Career construction theory and practice. In D. Brown and R. W. Lent (Eds) Career development and counseling: Putting theory and research to work (2nd ed, pp.147-183). Hoboken, NJ: John Wiley and Sons.

Savickas, M. L., Nota, L., Rossier, J., Dauwalder, J-P., Duarte, M. E.,... Guichard, J. (2009). Life designing: A paradigm for career construction in the 21st century. Journal of Vocational Behavior, 75(3), 239-250. doi: 10.1016/j.jvb.2009.04.004

Savickas, M. L., Nota, L., Rossier, J., Dauwalder, J.-P., Duarte, M. E., Guichard, J., Soresi, S., Van Esbroeck, R., \& van Vianen, A. E. M. (2010/2011). A construção da vida: Um novo paradigma para compreender a carreira no século XXI. Revista Portuguesa de Psicologia, 42, 13-4.

Savickas, M. L., \& Porfeli, E. J. (2012). Career Adapt-Abilities Scale: Construction, reliability, and measurement equivalence across 13 countries. Journal of Vocational Behavior, 80(3), 661-673. doi: 10.1016/j.jvb.2012.01.011

Tolentino, L., Garcia, P., Lu, V., Restubog, S., Bordia, P., \& Plewa, C. (2014). Career adaptation: The relation of adaprability to goal orientation, proactive personality, and career optimism. Journal of Vocational Behavior, 84(1), 39-48. doi: 10.1016/j.jvb.2013.11.004

Van Esbroeck, R. (2008). Career guidance in a global world. Em J. A. Athanasou \& R. V. Esbroeck (Eds.), International Handbook of Career Guidance (pp. 23-44). New York: Springer.

\section{Sobre a autora}

Alexandra Barros é professora, investigadora e subdiretora da Faculdade de Psicologia da Universidade de Lisboa, especialista em Psicologia da Educação e em Psicologia das Organizações e Recursos Humanos, com várias publicações em revistas internacionais. 